http://dx.doi.org/10.1590/1678-7660

Arq. Bras. Med. Vet. Zootec., v.67, n.2, p.547-554, 2015

\title{
Toxicidade aguda ao sal comum e larvicultura intensiva do jundiá Rhamdia quelen em água salobra
}

\author{
[Acute toxicity of common salt and intensive larviculture of silver catfish \\ Rhamdia quelen in brackish water] \\ T.E.H.P. Fabregat ${ }^{1}$, J. Damian ${ }^{1}$, N.S. Fialho ${ }^{1}$, D. Costa $^{1}$, J.A. Broggi ${ }^{1}$, \\ R.G. Pereira', R. Takata \\ ${ }^{1}$ Universidade do Estado de Santa Catarina, Centro de Ciências Agroveterinárias - Lages, SC \\ ${ }^{2}$ Fundação Instituto de Pesca do Estado do Rio de Janeiro - RJ
}

\begin{abstract}
RESUMO
A tolerância de peixes de água doce à salinidade e os níveis adequados de náuplios de Artemia na alimentação durante a larvicultura são de extrema importância para a padronização dos manejos em ambientes de criação intensiva. Dessa forma, o objetivo do trabalho foi estimar a salinidade letal $\left(\mathrm{SL}_{50}\right)$ para larvas de jundiá Rhamdia quelen e determinar o efeito da salinidade e da concentração de presas vivas na larvicultura intensiva. No primeiro ensaio, larvas ao final do período lecitotrófico $(1,1 \pm 0,8 \mathrm{mg})$ foram submetidas às salinidades de $0,2,4,6,8,10,15$ e $20 \mathrm{~g}$ de sal/L por um período de $96 \mathrm{~h}$. No segundo experimento, as larvas de jundiá, no início da alimentação exógena $(1,2 \pm 0,3 \mathrm{mg})$, foram submetidas a três salinidades (água doce 0,2 e 4g de sal/L) e três concentrações de presas vivas (início: 300, 500, 700 náuplios de Artemia/larvas/dia, sendo esse montante aumentado a cada cinco dias). $\mathrm{O}$ experimento foi realizado em delineamento inteiramente ao acaso, em esquema fatorial 3x3, por um período de 15 dias. No experimento 1, as larvas de jundiá submetidas às salinidades de 10,15 e $20 \mathrm{~g}$ de sal/L morreram após 12, duas e uma hora de exposição, respectivamente. As $\mathrm{SL}_{50}$ de 72 e $96 \mathrm{~h}$ foram estimadas em 9,93 e $4,95 \mathrm{~g}$ de sal/L, respectivamente. No final do teste de toxicidade, não houve diferença na sobrevivência entre as salinidades de 0,2 e $4 \mathrm{~g}$ de sal/L. No experimento 2, não foi observado efeito da interação entre salinidade e concentração de presas para o peso e o comprimento. Quanto maior a quantidade de presas, maior o crescimento das larvas. A sobrevivência apresentou interação entre os fatores. O aumento da salinidade proporcionou uma diminuição da sobrevivência, independentemente da concentração de presas. Dessa forma, conclui-se que a $\mathrm{SL}_{50}$ diminuiu com o aumento do tempo de exposição à água salinizada e que a larvicultura da espécie pode ser realizada em salinidades de até $2 \mathrm{~g}$ de sal/L, com concentração de presas vivas diária inicial de 700 náuplios de Artemia/larva.
\end{abstract}

Palavras-chave: alimento vivo, bagre, piscicultura, salinidade

\begin{abstract}
The tolerance of freshwater fish to salinity and the adequate levels of Artemia nauplii in the feeding regime during larviculture are of extreme importance to the standardization of management practices in intensive production environments. Therefore, the aim of this study was to estimate the lethal salinity $\left(L S_{50}\right)$ of the silver catfish Rhamdia quelen larvae and determine the effect of salinity and the concentrations of live prey in intensive larviculture of this species. In the first trial, larvae at the end of the lecithotrophic period $(1.1 \pm 0.8 \mathrm{mg})$ were subjected to salinities of $0,2,4,6,8,10,15$ and $20 \mathrm{~g}$ of salt/L for a period of $96 \mathrm{~h}$. In the second experiment, the catfish larvae starting the exogenous feed $(1.2 \pm 0.3 \mathrm{mg})$ were subjected to three salinities (freshwater 0,2 and $4 \mathrm{~g}$ of salt/L) and three concentrations of live prey (starting at: 300, 500, 700 Artemia nauplii/larvae/day, this amount being increased every five days). The experiment was conducted in a completely randomized design in a $3 \times 3$ factorial scheme, for a period of 15 days. In experiment 1, the catfish larvae subjected to the salinities of 10, 15 and $20 \mathrm{~g}$ of salt/L died
\end{abstract}

Recebido em 16 de maio de 2014

Aceito em 9 de janeiro de 2015

E-mail: thiagofabregat@hotmail.com 
after 12, 2 and 1 h of exposure, respectively. The LS50 at 72 and $96 \mathrm{~h}$ were estimated at 9.93 and $4.95 \mathrm{~g}$ of salt/L, respectively. At the end of the toxicity test, there was no difference in the survival among the salinities of 0,2 and $4 \mathrm{~g}$ of salt/L. In experiment 2 no significant interaction was observed between salinity and the concentration of prey for weight and length. The increased quantity of prey increased the growth of the larvae. The rise in the salinity correlated to a decrease in survival, regardless of the prey concentration. Thus, it is concluded that the LS5O decreased with the increase in time of exposure to saltwater, and that the larviculture of this specie can be conducted in salinities of up to $2 \mathrm{~g}$ salt/L, with a daily prey concentration starting at 700 Artemia/larvae.

Keywords: catfish, live food, fish farming, salinity

\section{INTRODUÇÃO}

A larvicultura de peixes neotropicais é um assunto que está em pauta no cenário científico internacional (Jomori et al., 2012; Melillo-Filho et al., 2014; Portella et al., 2014). As larvas das espécies reofílicas de interesse comercial são altriciais, que possuem reserva vitelínica reduzida e cujo trato digestório não está totalmente desenvolvido no início da alimentação exógena (revisado por Portella et al., 2014). Nessa fase as larvas precisam ser alimentadas com alimento vivo, e o manejo na larvicultura intensiva é complexo. Assim, é necessário o desenvolvimento de técnicas para otimizar o cultivo das larvas no laboratório e maximizar os resultados produtivos (Jomori et al., 2003).

A água salinizada auxilia a fase inicial de desenvolvimento das larvas, pois ajuda a driblar as perdas de íons e a excessiva absorção de água, consequência das diferenças osmóticas entre o plasma dos peixes e o meio externo (Varsamos et al., 2005). O sal pode ser utilizado para prevenir doenças (Marchioro e Baldisserotto, 1999; Souza-Bastos e Freire, 2009), promover o crescimento e/ou a sobrevivência (Luz e Portella, 2002; Beux e Zaniboni-Filho, 2007; Santos e Luz, 2009) e reduzir o estresse (Wurts, 1995). Além disso, meios ligeiramente salinizados otimizam o aproveitamento dos náuplios de Artemia (Lopes et al., 1996; Jomori et al. 2012), microcrustáceo de ambiente salino e um dos principais alimentos vivos fornecidos durante a larvicultura intensiva (Luz e Portella, 2002; Kaiser et al., 2003; Jomori et al., 2003; Fabregat et al., 2008; Santos e Luz, 2009; Luz e Santos, 2010; Jomori et al,. 2013).

O jundiá Rhamdia quelen é de grande importância para a região Sul do Brasil, por se adaptar bem a diferentes ambientes, ser amplamente difundida nos viveiros de piscicultura e por ser bem aceito pelo mercado consumidor (Baldisseroto e Radünz-Neto, 2004). As larvas de jundiá normalmente são criadas por alguns dias no laboratório antes de serem soltas em viveiros externos, mas alguns estudos foram realizados para viabilizar o cultivo inicial das larvas em sistema intensivo (Piaia e RadünzNeto, 1997; Behr et al., 1999; Salhi e Bessonart, 2011; Diemer et al., 2012). O jundiá consegue ingerir dieta formulada desde o início da alimentação exógena (Borges-Neto et al, 2013), mas o alimento vivo em combinação com a dieta formulada pode ser utilizado para incrementar o desempenho e a sobrevivência, como observado para demais espécies de água doce (Kaiser et al., 2003; Diemer et al., 2012). Os náuplios de Artemia são uma excelente alternativa como alimento para as larvas de jundiá criadas em condições intensivas. Assim, o objetivo deste estudo foi determinar a concentração letal de sal $\left(\mathrm{SL}_{50}\right)$ para larvas de jundiá, bem como a concentração de presas que deve ser fornecida para larvas em sistema de larvicultura intensiva.

\section{MATERIAL E MÉTODOS}

Foram conduzidos dois experimentos no Laboratório de Piscicultura, do Centro de Ciências Agroveterinárias da Universidade do Estado de Santa Catarina - CAV/UDESC, Lages, Santa Catarina. Os experimentos foram aprovados pelo comitê de ética da universidade, com o número do protocolo 1.27.13.

Para determinar a faixa letal (SL50) de concentração salina, foi conduzido um teste de tolerância à salinidade. Larvas de jundiá com três dias após a eclosão, na fase inicial da alimentação exógena $(1,1 \pm 0,8 \mathrm{mg})$, foram submetidas a diferentes concentrações salinas ( $\mathrm{NaCl}$ comum): 0, 2, 4, 6, 8, 10, 15 e $20 \mathrm{~g}$ de sal/L, por um período de 96 horas. O 
experimento foi realizado em aquários de $1 \mathrm{~L}$ de volume útil, nos quais as larvas foram estocadas na densidade de 10 larvas por litro e mantidas em jejum, sob temperatura e aeração constante durante toda a avaliação. O delineamento foi inteiramente ao acaso, composto por oito tratamentos (salinidades) e seis réplicas. A mortalidade foi avaliada a cada hora, nas primeiras 24 horas e, posteriormente, a cada 24 horas.

O experimento foi conduzido em sistema semiestático, em que $100 \%$ da água foi renovada a cada 24 horas. Durante a renovação, as larvas eram separadas com uma peneira (malha $1 \mathrm{~mm}$ ) e os aquários eram limpos. A temperatura média (termômetro de álcool) foi aferida diariamente, antes das trocas de água. $\mathrm{O} \mathrm{pH}$ (phmetro Lutron modelo PH-221), o oxigênio dissolvido (oxímetro Lutron modelo DO-5519) e a concentração de amônia total (Eaton et al., 2005) foram aferidos a cada sete dias. Os valores (temperatura $26,3^{\circ} \mathrm{C} \pm 1,06 ; \quad \mathrm{pH} \quad 8,42 \pm 0,16$; oxigênio dissolvido $7,46 \pm 0,65 \mathrm{mg} / \mathrm{L}$ e amônia $0,25 \mathrm{ppm}$ ) mantiveram-se dentro do limite adequado para cultivo do jundiá (Baldisseroto e Radünz-Neto, 2004). Para obtenção das salinidades desejadas, o sal ( $\mathrm{NaCl}$ comum) foi pesado, misturado à água e, posteriormente, a salinização foi confirmada com um refratômetro portátil (Biobrix modelo 211).

Ao completarem-se as 96 horas de experimento, as larvas sobreviventes foram quantificadas. As médias obtidas foram submetidas ao teste de ANOVA e, quando houve diferença significativa, foi realizada comparação pelo teste de Tukey. Todos os dados foram submetidos a testes para a verificação da normalidade dos erros e da homocedasticidade das variâncias. A salinidade letal $\left(\mathrm{SL}_{50}\right)$ em $96 \mathrm{~h}$ foi calculada por meio do programa "Trimmed Spearman Karber method".

O experimento 2 foi conduzido para se estudar a relação entre a salinidade e o aproveitamento do alimento vivo. Larvas de jundiá com três dias após a eclosão, na fase inicial da alimentação exógena $(1,2 \pm 0,3 \mathrm{mg})$, foram criadas em diferentes concentrações salinas, preestabelecidas no experimento $1(0,2 \mathrm{e}$ $4 \mathrm{~g}$ de sal/L), e cada uma delas foi testada com três concentrações de presas (inicialmente: 300, 500, 700 náuplios de Artemia/larvas/dia), durante
15 dias. As larvas foram distribuídas em aquários experimentais de volume útil de $1 \mathrm{~L}$, na densidade inicial de 10 larvas por litro. O delineamento experimental foi inteiramente ao acaso, em esquema fatorial 3 x 3 , com três salinidades e três concentrações de presas, com seis repetições cada, totalizando 54 unidades experimentais. Os cistos de Artemia foram incubados diariamente para obtenção dos náuplios recém-eclodidos. $\mathrm{O}$ controle da alimentação foi feito estimando-se o número de náuplios por mililitro do meio em que eram mantidos. Os níveis de alimentação mencionados foram determinados com base em ensaios-piloto realizados no laboratório. As quantidades fornecidas foram aumentadas a cada cinco dias, quando quantidades iguais às iniciais foram acrescentadas em cada tratamento.

O experimento foi realizado em temperatura constante $\left(25^{\circ} \mathrm{C}\right)$, mantida por meio de banhos termostatizados, e os aquários foram suplementados com aeração artificial oriunda de compressor de ar. Diariamente, cerca de $80 \%$ da água era renovada e eram retirados os restos de alimentos e verificada a presença de larvas mortas. Os parâmetros físicos e químicos da água se mantiveram dentro do limite adequado para o cultivo do jundiá (Baldisseroto e Radünz-Neto, 2004) e apresentaram médias de temperatura $25,5^{\circ} \mathrm{C} \pm 0,57 ; \mathrm{pH} 8,52 \pm 0,15$; oxigênio dissolvido $7,03 \pm 1,42 \mathrm{mg} / \mathrm{L}$ e amônia total $0,89 \pm 0,22 \mathrm{ppm}$. Para obtenção das salinidades desejadas, o sal foi pesado, misturado à água e, posteriormente, a salinização foi confirmada com um refratômetro portátil.

As biometrias foram realizadas no início e ao final do experimento (15 dias). As larvas sobreviventes foram eutanasiadas com uma overdose de eugenol, conservadas em formol $10 \%$ tamponado e, após 24 horas, transferidas para solução de álcool $70 \%$. O comprimento total das larvas foi medido com um paquímetro digital $(\mathrm{mm})$ e o peso foi aferido em balança analítica de precisão. Ao final do experimento, foi calculada a taxa de sobrevivência dos tratamentos. Os resultados foram analisados estatisticamente por meio de análise de variância paramétrica (ANOVA) e análise de regressão. Quando houve diferenças significativas, as médias foram comparadas pelo teste de Tukey. Todos os dados foram submetidos a testes para a verificação da normalidade dos erros e da homocedasticidade 
das variâncias. Os dados de sobrevivência sofreram transformação arcoseno antes de serem analisados.

\section{RESULTADOS E DISCUSSÃO}

No teste de tolerância à salinidade, as larvas de jundiá expostas à salinidade máxima $(20 \mathrm{~g}$ de sal/L) não resistiram à primeira hora de experimento. Já na concentração de $15 \mathrm{~g}$ de sal/L, a sobrevivência das larvas não passou da segunda hora de experimento. $\mathrm{Na}$ concentração de $10 \mathrm{~g}$ de sal/L, a letalidade máxima foi observada após 12 horas. Na Tab. 1 estão apresentados os dados de sobrevivência observada nos demais tratamentos, a partir das primeiras 12 horas de avaliação. Somente a partir de 72 horas foi observada diferença estatística entre os tratamentos. Nesse período, apenas as larvas submetidas à salinidade de $8 \mathrm{~g}$ de sal/L apresentaram menor sobrevivência $(\mathrm{P}<0,05) \mathrm{em}$ relação às outras salinidades. $\mathrm{A} \mathrm{SL}_{50}$ após 72 horas foi calculada em $9,93 \mathrm{~g}$ de sal/L (intervalo de confiança: 9,51-10,23) e os dados de sobrevivência foram expressos pela equação $\mathrm{x}=$ $-1,5536 x^{2}+8,1786 x+94,971\left(R^{2}=0,90\right)$, com concentração salina ótima estimada em $2,63 \mathrm{~g}$ de sal/L. Após 96 horas, a sobrevivência foi significativamente maior $(\mathrm{P}<0,05)$ nas larvas mantidas na salinidade de até $4 \mathrm{~g}$ de sal/L, não diferindo das larvas mantidas em água doce. A $\mathrm{SL}_{50}$ após 96 horas foi calculada em 4,95g de sal/L (intervalo de confiança: 4,62-5,09) e os dados de sobrevivência foram expressos pela equação $x=-1,2679 x^{2}-2,1071 x+100,26\left(R^{2}=\right.$ $0,90)$, com concentração salina ótima estimada

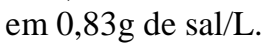

Tabela 1. Sobrevivência (\%) de larvas de jundiá submetidas a diferentes gradientes de salinidade por 96 horas

\begin{tabular}{lccccc}
$\mathrm{g} \mathrm{de} \mathrm{sal} / \mathrm{L}$ & $12 \mathrm{~h}$ & $24 \mathrm{~h}$ & $48 \mathrm{~h}$ & $72 \mathrm{~h}$ & $96 \mathrm{~h}$ \\
\hline 0 & $98,0 \pm 0,4 \mathrm{a}$ & $98,0 \pm 0,4^{\mathrm{a}}$ & $98,0 \pm 0,4 \mathrm{a}$ & $98,0 \pm 0,4 \mathrm{a}$ & $98,0 \pm 0,4 \mathrm{a}$ \\
2 & $100,0 \pm 0,0 \mathrm{a}$ & $100,0 \pm 0,0 \mathrm{a}$ & $100,0 \pm 0,0 \mathrm{a}$ & $100,0 \pm 0,0 \mathrm{a}$ & $93,0 \pm 1,3 \mathrm{a}$ \\
4 & $100,0 \pm 0,0 \mathrm{a}$ & $100,0 \pm 0,0 \mathrm{a}$ & $100,0 \pm 0,0 \mathrm{a}$ & $100,0 \pm 0,0 \mathrm{a}$ & $79,0 \pm 1,7 \mathrm{a}$ \\
6 & $100,0 \pm 0,0 \mathrm{a}$ & $100,0 \pm 0,0 \mathrm{a}$ & $100,0 \pm 0,0 \mathrm{a}$ & $97,0 \pm 2,2 \mathrm{a}$ & $30,0 \pm 2,3 \mathrm{~b}$ \\
8 & $100,0 \pm 0,0 \mathrm{a}$ & $97,0 \pm 0,5^{\mathrm{a}}$ & $97,0 \pm 0,5 \mathrm{a}$ & $57,0 \pm 1,4 \mathrm{~b}$ & $7,0 \pm 1,1 \mathrm{c}$ \\
\hline $\mathrm{CV} \%$ & 1,90 & 3,18 & 3,18 & 25,58 & 67,50
\end{tabular}

Médias seguidas da mesma letra na vertical não diferem entre si pelo teste de Tukey $(\mathrm{P}>0,05)$.

Os resultados apontaram que as larvas de jundiá não suportaram salinidades elevadas, acima de $10 \mathrm{~g}$ de sal/L, mesmo quando expostas em um curto intervalo de tempo. Em salinidades menores, até $8 \mathrm{~g}$ de sal/L, a exposição durante 48 horas não afetou a sobrevivência. Esse resultado é relevante, pois os banhos concentrados de sal são muito eficientes no tratamento de ectoparasitas como o Ichthyophthirius multifilii (Garcia et al., 2007; Souza-Bastos e Freire, 2009). Os juvenis de jundiá toleraram salinidades maiores, de até $9 \mathrm{~g}$ de sal/L, sem prejuízo para a sobrevivência (Marchioro e Baldisserotto, 1999). De maneira geral, muitas espécies de água doce conseguem se desenvolver em salinidades acima de $9 \mathrm{~g}$ de sal/L (Kasim, 1983). A diferença no desenvolvimento ontogênico pode explicar por que os juvenis e os peixes maiores são mais tolerantes à salinidade do que as larvas (Luz e Santos, 2008). Após a eclosão e durante o início do estágio larval, a osmorregulação é realizada pelo epitélio; posteriormente essa função é transferida para as brânquias, que passam a ser o principal órgão com função osmorregulatória (Varsamos et al., 2005).

As larvas de jundiá sobreviveram bem na água ligeiramente salinizada ( $2 \mathrm{~g}$ de sal/L), e essa concentração pode ser utilizada durante a larvicultura. Essa observação pode ser comprovada por meio dos resultados de sobrevivência e dos cálculos de concentração salina ótima com 72 e 96 horas. Resultados similares também foram obtidos com larvas de acará-bandeira (Pterophyllum scalare), em que a sobrevivência foi maior até às 96 horas no gradiente de menor concentração ( $2 \mathrm{~g}$ de sal/L) e em água doce (Fabregat et al., 2008). Além disso, já foi demonstrado que esse nível de salinização proporciona bons resultados de 
crescimento e sobrevivência na larvicultura de outras espécies de peixes em comparação com a água doce (Santos e Luz, 2009; Jomori et al., 2012; Luz et al., 2012; Jomori et al., 2013). Por outro lado, as larvas de pacamã (Lophiosilurus alexandri) toleraram salinidades de $6 \mathrm{~g}$ de sal/L por até 96 horas (Santos e Luz, 2008).

A diminuição nos valores obtidos de $\mathrm{SL}_{50}$ entre os tempos de 72 e 96 horas, a qual passou de 9,93 para $4,95 \mathrm{~g}$ de $\mathrm{sal} / \mathrm{L}$, é resultado da diminuição da sobrevivência nas maiores salinidades. Resultados semelhantes foram observados para larvas de bagre africano Clarias gariepinus, em que a $\mathrm{SL}_{50}$ para os tempos de 49,5 e 59 horas diminuiu de 10 para $2 \mathrm{~g}$ de sal/L (Gbulubo et al., 1998). Por outro lado, para larvas recém-eclodidas de outra espécie de bagre africano Heterobranchus longifilis, a $\mathrm{SL}_{50}$ após 96 horas foi de 4,35g de sal/L (Fashina-Bombata e Busari, 2003). Em larvas de pacamã com oito dias após a eclosão, a SL 50 após 96 horas foi de 8,94g de sal/L (Luz e Santos, 2008). Essa diferença pode estar relacionada à fase de desenvolvimento dos animais, como já mencionado, e à própria fisiologia da espécie, o que levaria a valores diferenciados de toxicidade ao sal, como a $\mathrm{SL}_{50}$ de $21,5 \mathrm{~g}$ de sal/L para larvas de tilápia híbrida $O$. niloticus $x O$. aureus com sete dias após a eclosão (Watanabe et al., 1985).

No experimento 2, não foi observada interação entre a salinidade e a concentração de presas $(\mathrm{P}>0,05)$ para a variável ganho em peso (Tab. 2). A utilização de água salinizada em 2 e $4 \mathrm{~g}$ de sal/L não afetou $(\mathrm{P}>0,05)$ o ganho em peso das larvas. A salinização da água já se mostrou eficiente para maximizar os resultados da larvicultura de espécies de peixes neotropicais alimentadas com náuplios de Artemia (Santos e Luz, 2009; Jomori et al., 2012; Jomori et al., 2013). Entretanto, no presente estudo, não foi observado efeito da salinidade da água sobre o crescimento das larvas de jundiá. Outros autores também não encontraram efeito da água salinizada em 2 e $4 \mathrm{~g}$ de $\mathrm{sal} / \mathrm{L}$ sobre o desempenho de larvas de trairão Hoplias lacerdae, pintado Pseudoplatystoma corruscans, curimbatá Prochilodus costatus e pacamã (Luz e
Portella, 2002; Santos e Luz, 2009). Uma hipótese para a falta de diferenças observada em alguns estudos está relacionada com a quantidade de alimento fornecida. A salinização da água prolonga a sobrevivência dos náuplios, deixando estes mais tempo disponíveis para serem ingeridos pelas larvas (Beux e Zaniboni-Filho, 2007; Jomori et al., 2012). Assim, para que o efeito da água salinizada seja mais evidente, é necessário que haja sobra de náuplios após a alimentação (Luz e Portella, 2002; Beux e Zaniboni-Filho, 2007). No presente estudo, essa possibilidade pode ser descartada, pois havia sobra de alimento nos aquários no final do dia, principalmente quando a concentração de presas era aumentada. Futuros estudos serão realizados para otimizar a relação entre a salinidade e a concentração de presas durante a alimentação inicial de larvas de jundiá, o que fará com que o manejo e o comportamento alimentar da espécie sejam melhor compreendidos.

O aumento da concentração de presas foi proporcional $(\mathrm{P}<0,05)$ ao aumento no ganho de peso, e os melhores resultados foram obtidos com o fornecimento inicial de 700 náuplios/larva. Não foi encontrado, na literatura, nenhum estudo quantificando o número de náuplios de Artemia para a alimentação de larvas de jundiá, mas a sua eficácia nesse período já foi comprovada (Diemer et al., 2012). Para larvas de outras espécies de peixes, as concentrações de náuplios fornecidas variam bastante, porém, em geral, são próximas das que foram fornecidas para as larvas de jundiá. Na larvicultura do pintado, outra espécie de bagre, as recomendações iniciais variam entre 300 e 500 náuplios/larva (Beux e Zaniboni-Filho, 2007; Santos e Luz, 2009). Para o trairão, foram definidos 300 náuplios/larva. (Luz e Portella, 2002). Para o bocudo (Steindachneridion scripta), foram utilizadas menores concentrações de presas, 100 náuplios/larva (Adamante et al., 2007), assim como para o suruvi (Steindachneridion scriptum) (Schütz et al., 2008). Outras espécies podem necessitar de concentrações ainda mais altas, como o pacamã, cuja recomendação inicial é em torno de 1000 náuplios/larva (Melillo-Filho et al., 2014). 
Tabela 2. Análise de variância e valores médios das variáveis de desempenho de larvas de jundiá ao final do experimento 2

\begin{tabular}{|c|c|c|c|}
\hline Estatística & $\begin{array}{l}\text { Peso final } \\
\quad(\mathrm{mg})\end{array}$ & $\begin{array}{l}\text { Comprimento total } \\
\qquad(\mathrm{mm})\end{array}$ & $\begin{array}{c}\text { Sobrevivência } \\
(\%)\end{array}$ \\
\hline \multicolumn{4}{|l|}{ Valores de F } \\
\hline Efeito da salinidade (S) & $0,47^{\mathrm{ns}}$ & $0,33^{\mathrm{ns}}$ & $9,02^{* *}$ \\
\hline Efeito dos níveis (N) & $88,11^{* *}$ & $121,66^{* *}$ & $0,27^{\mathrm{ns}}$ \\
\hline Interação (S x N) & $1,34^{\mathrm{ns}}$ & $0,33^{\mathrm{ns}}$ & $3,63^{*}$ \\
\hline $\mathrm{CV}(\%)$ & 14,56 & 4,04 & 11,46 \\
\hline \multicolumn{4}{|c|}{ Médias para as salinidades (g de sal/L) } \\
\hline 0 & $69,79 \pm 20,61$ & $19,08 \pm 1,75$ & $80,55 \pm 12,11 \mathrm{a}$ \\
\hline 2 & $67,99 \pm 20,18$ & $19,50 \pm 2,10$ & $67,77 \pm 14,37 \mathrm{~b}$ \\
\hline 4 & $66,60 \pm 21,91$ & $19,26 \pm 1,82$ & $65,55 \pm 10,41 b$ \\
\hline \multicolumn{4}{|c|}{ Médias para as concentrações de presa (náuplios de Artemia/larva) } \\
\hline 300 & $44,58 \pm 7,40 \mathrm{c}$ & $17,05 \pm 0,74 \mathrm{c}$ & $71,11 \pm 21,70$ \\
\hline 500 & $71,75 \pm 11,75 b$ & $19,56 \pm 0,98 b$ & $70,00 \pm 11,88$ \\
\hline 700 & $88,05 \pm 10,20 \mathrm{a}$ & $21,03 \pm 0,62^{\mathrm{a}}$ & $72,77 \pm 11,78$ \\
\hline
\end{tabular}

Médias seguidas de mesma letra minúsculas iguais na vertical não apresentam diferenças significativas pelo teste de Tukey. *: $\mathrm{P}<0,05 ; * *$ : $\mathrm{P}<0,01 ;{ }^{\text {ns; }}$, não significativo.

Para a sobrevivência, houve interação significativa $(\mathrm{P}<0,05)$ entre a salinidade e o nível de alimentação. $\mathrm{O}$ desdobramento da interação (Tab. 3) mostrou que, nas menores concentrações de presas, 300 e 500 náuplios/larva, a sobrevivência das larvas na água doce foi maior $(\mathrm{P}<0,05)$ do que na água salinizada. Com o fornecimento de 700 náuplios/larva, a sobrevivência foi mais alta na água doce e na água salinizada em $2 \mathrm{~g}$ de sal/L. De maneira geral, é esperado aumento da sobrevivência das larvas com a utilização de água salinizada (Beux e Zaniboni-Filho, 2007; Luz e Santos, 2008), entretanto, no presente estudo, foi observado o contrário. Embora o jundiá seja um peixe que é encontrado em ambientes de estuário (Marchioro e Baldisserotto, 1999), na fase larval a exposição prolongada à água salinizada prejudicou a sobrevivência dos animais. No nível mais alto de alimentação, a salinização em $2 \mathrm{~g}$ de sal/L não prejudicou a sobrevivência, mas, como observado anteriormente, também não houve o beneficio esperado sobre o crescimento com a maior disponibilidade de náuplios, o que pode indicar um possível efeito negativo da salinização sobre o desempenho.

Tabela 3. Desdobramento da interação dos valores de sobrevivência de larvas de jundiá após 15 dias de experimento

\begin{tabular}{lccc}
\hline $\begin{array}{l}\text { Concentração de presas } \\
\text { (náuplios/larva) }\end{array}$ & 0 & 2 & \multicolumn{3}{c}{ Salinidade (g de sal/L) } \\
\cline { 2 - 4 } & $90,00 \pm 10,95 \mathrm{Aa}$ & $60,00 \pm 10,95 \mathrm{Ba}$ & $63,33 \pm 13,66 \mathrm{Ba}$ \\
500 & $76,66 \pm 8,16 \mathrm{Aa}$ & $65,00 \pm 16,43 \mathrm{Ba}$ & $68,33 \pm 7,52 \mathrm{Ba}$ \\
700 & $75,00 \pm 12,24 \mathrm{Aa}$ & $78,33 \pm 9,83 \mathrm{Aa}$ & $65,00 \pm 10,48 \mathrm{Ba}$ \\
\hline CV $(\%)$ & & 20,77
\end{tabular}

Médias seguidas da mesma letra (maiúscula na horizontal em cada subquadro e minúscula na vertical) não diferem entre si pelo teste de Tukey $(\mathrm{P}>0,05)$.

\section{CONCLUSÕES}

A $\mathrm{SL}_{50}$ das larvas diminui com o aumento do tempo de exposição à água salinizada. A larvicultura do jundiá pode ser realizada em salinidades de até $2 \mathrm{~g}$ de sal/L, com concentração inicial de presas inicial de 700 náuplios de Artemia/larva. 


\section{REFERÊNCIAS}

ADAMANTE, W.B.; WEINGARTNER, M.; NUÑER, A.P.O. Feed transition in larval rearing of bocudo, Steindachneridion scripta (Pisces, Pimelodidae), using Artemia spp. nauplii and artificial diet. Arq. Bras. Med. Vet. Zootec., v.59, p.1294-1300, 2007.

BALDISSEROTO, B.; RADÜNZ-NETO, J. Criação de jundiá. Santa Maria: UFSM, 2004, 232p.

BEHR, E.R.; NETO, J.R.; TRONCO, A.P.; FONTANA, A. Influência de diferentes níveis de luminosidade sobre o desempenho de larvas de jundiá (Rhamdia quelen). Acta Sci. Anim. Sci., v.21, p.323-333, 1999.

BEUX, L.F.; ZANIBONI FILHO, E. Survival and the growth of pintado (Pseudoplatystoma corruscans) post-larvae on different salinities. Braz. Arch. Biol. Technol., v.50, p.821-829, 2007

BORGES-NETO, P.G.; DUTRA, F.M.; BALLESTER, E.L.C.; PORTZ, L. Crescimento e sobrevivência de larvas do jundiá, Rhamdia quelen, alimentadas com alimento vivo enriquecido e dieta artificial. Rev. Bras. Cienc. Vet., v.216-221, p.20, 2013.

DIEMER, O.; NEU, D.H.; SARY, C. et al. Artemia sp. na alimentação de larvas de jundiá (Rhamdia quelen). Cienc. Anim. Bras., v.13, p.175-179, 2012.

EATON, A.D.; CLESCERI, L.S.; RICE, E.W.; GREENBERG, A.E. Standard methods for the examination of water and wastewater. Washington: American Public Health Association, 2005. 1325 p.

FABREGAT, T.E.H.P.; FERNANDES, J.B.K.; TIMPONE, I.T. et al. Utilização de água salinizada e náuplios de Artemia durante a larvicultura do acará-bandeira Pterophyllum scalare. In: CYRINO, J.E.P.; SCORVO-FILHO, J.D.; SAMPAIO, L.A.; CAVALLI, R.O. (Eds.) Tópicos especiais em biologia aquática $e$ aquicultura, Piracicaba-SP: Copiadora Luiz de Queiroz, 2008.p.105-110.

FASHINA-BOMBATA, H.A.; BUSARI, A.N. Influence of salinity on the developmental stages of African catfish Heteobranchus longifilis (Valenciennes, 1840). Aquaculture, v.224, p.213$222,2003$.
GARCIA, L.O.; BECKER, A.G.; COPATTI, C.E.; BALDISSEROTTO, B. Salt in the food and water as a supportive therapy for Ichthyophthirius multifilii infestation on silver catfish Rhamdia quelen fingerlings. J. World Aquaculture Soc., v.38, p.1-11, 2007.

GBULUBO, A.J.; ERONDU, E.S. Salinity influence on the early stages of the african catfish. Aquacul. Internat., v.6, p.369-379, 1998.

JOMORI, R.K.; CARNEIRO, D.J.; MALHEIROS, E.B.; PORTELLA, M.C. Growth and survival of pacu Piaractus mesopotamicus (Holmberg,1887) juveniles reared in ponds or at different initial larviculture periods indoors. Aquaculture, v.221, p.277-287, 2003.

JOMORI, R.K.; LUZ, R.K.; PORTELLA, M.C. Effect of salinity on larval rearing of pacu, Piaractus mesopotamicus, a Freshwater Species. J. World Aquaculture Soc., v.43, p.423-432, 2012.

JOMORI, R.K.; LUZ, R.K.; TAKATA, R. et al. Água levemente salinizada aumenta a eficiência da larvicultura de peixes neotropicais. Pesq. Agropec. Bras., v.48, p.809-815, 2013.

KAISER, H.; ENDEMANN, F.; PAULET, T.G. A comparison of artificial and natural foods and their combinations in the rearing of goldfish, Carassius auratus (L.). Aquacul. Research, v.34, p.943-950, 2003.

KASIM, H.M. Salinity tolerance of fresh water fishes. Indian J. Fish., v.30, p.46-54, 1983.

LOPES, R.N.M.; FREIRE, R.A.B.; VICENSOTTO, J.R.M. et al. Alimentação de larvas de surubim Peseudoplatystoma corruscans (AGASSIZ, 1829) em laboratório na primeira semana de vida. Bol. Tec. CEPTA, v.9, p.11-29, 1996.

LUZ, R.K.; PORTELLA, M.C. Larvicultura de trairão (Hoplias lacerdae) em água doce e água salinizada. Rev. Bras. Zootec., v.31, p.829-834, 2002.

LUZ, R.K.; SANTOS, J.C.E. Avaliação da tolerância de larvas de pacamã Lophiosilurus alexandri Steindachner, 1877 (Pisces: Siluriformes) a diferentes salinidades. Acta Sci. Biol. Sci., v.30, p.345-350, 2008. 
LUZ, R.K.; SANTOS, J.C.E. Effect of salt addition and feeding frequency on cascudo preto Rhinelepis aspera (Pisces: Loricariidae) larviculture. J. Appl. Ichthyol., v.26, p.453-455, 2010.

LUZ, R.K.; SILVA, W.; S.S.; MELILLOFILHO, R. et al. Stocking density in the larviculture of Nile tilapia in saline water. Rev. Bras. Zootec., v.41, p.2385-2389, 2012.

MARCHIORO, M.I.; BALDISSEROTTO, B. Sobrevivência de alevinos de jundiá (Rhamdia quelen quoy \& gaimard, 1824) à variação de salinidade da água. Cienc. Rural, v.29, p.315$318,1999$.

MELILLO-FILHO, R.; TAKATA, R.; SANTOS, A.E.H. et al. Draining system and feeding rate during the initial development of Lophiosilurus alexandri (Steindachner, 1877), a carnivorous freshwater fish. Aquacul. Resea., v.45, p.19131920, 2014.

PIAIA, R.; RADÜNZ-NETO, J. Avaliação de diferentes fontes proteicas sobre o desempenho de larvas de jundiá (Rhamdia quelen). Cienc. Rural, v.27, p.319-323, 1997.

PORTELLA, M.C.; JOMORI, R.K.; LEITÃO, N.J. et al. Larval development of indigenous South American freshwater fish species, with particular reference to pacu (Piaractus mesopotamicus). Aquaculture, v.432, p.402-417, 2014.

SALHI, M.; BESSONART, M. Growth, survival and fatty acid composition of Rhamdia quelen (Quoy and Gaimard, 1824) larvae fed on artificial diet alone or in combination with Artemia nauplii. Aquacul. Resea., v.44, p.41-49, 2011.
SANTOS, J.C.E.; LUZ, R.K. Effect of salinity and prey concentrations on Pseudoplatystoma corruscans, Prochilodus costatus and Lophiosilurus alexandri larviculture. Aquaculture. v.287, p.324-328, 2009.

SCHÜTZ, J.H.; WEINGARTNER, M.; ZANIBONI-FILHO, E.; NUÑER, A.P.O. Crescimento e sobrevivência de larvas de suruvi Steindachneridion scriptum nos primeiros dias de vida: influência de diferentes alimentos e fotoperíodos. Boletim do Instituto de Pesca, v.34, p.443-451, 2008.

SOUZA-BASTOS, L.R.; FREIRE, C.A. The handling of salt by the neotropical cultured freshwater catfish Rhamdia quelen. Aquaculture. v.289, p.167-174, 2009.

VARSAMOS, S.; NEBEL, C.; CHAMANTIER, G. Ontogeny of osmoregulation in postembryonic fish: A review. Comp. Biochem. and Physiol., v.141, p.401-429, 2005.

WATANABE, W.O.; KUO, C-H.; HUANG, M.C. The ontogeny of salinity tolerance in the tilapias Oreochromis aureus, O. niloticus, and an $O$. mossambicus $\times O$. niloticus hybrid, spawned and reared in freshwater. Aquaculture, v.47, p.353-367, 1985.

WURTS, W.A. Using salt to reduce handling stress in channel catfish. World Aquaculture, v.26, p.80-81, 1995. 